\title{
EFECTO DEL ACEITE ESENCIAL DE Aloysia triphylla BRITTON (CEDRÓN) SOBRE EL Trypanosoma cruzi EN RATONES
}

\author{
Juan Rojas ${ }^{1, a}$, Olga Palacios ${ }^{2, b}$, Sergio Ronceros ${ }^{1, c}$
}

\begin{abstract}
RESUMEN
Objetivos. Determinar la actividad anti-Trypanosoma cruzi in vivo del aceite esencial de Aloysia triphylla en ratones. Materiales y Métodos. Los animales fueron asignados aleatoriamente a los siguientes grupos ( $\mathrm{n}=15$ por grupo): infectados y no tratados (G1), infectados y tratados con benznidazol $100 \mathrm{mg} / \mathrm{kg}(\mathrm{G} 2)$, infectados y tratados con aceite esencial de Aloysia triphylla $100 \mathrm{mg} / \mathrm{kg}$ (G3), infectados y tratados con aceite esencial de Aloysia triphylla $250 \mathrm{mg} / \mathrm{kg}$ (G4); no infectados y no tratados (G5), y no infectados y tratados con $250 \mathrm{mg} / \mathrm{kg}$ de Aloysia triphyla (G6). La infección con $T$. cruzi se realizó con $10^{4}$ tripomastigotes sanguíneos y el tratamiento empezó en el octavo día postinfección (dpi) hasta el 28 dpi. La parasitemia se determinó con microscopía óptica cada dos días en $5 \mu \mathrm{L}$ de sangre extraída de la cola. En el 14, 21 y 28 dpi se obtuvo sangre de la cola para el ensayo de creatina kinasa-MB (CK-MB), alanina aminotransferasa y creatinina; después, los animales fueron sacrificados y se extrajo el corazón para el estudio histopatológico. Resultados. El aceite esencial de cedrón produjo una reducción significativa de $85,4 \%$ del pico de parasitemia con la dosis de $250 \mathrm{mg} / \mathrm{kg}$; también produjo reducción del número de amastigotes e infiltrados inflamatorios en el corazón. El nivel plasmático de CK-MB también disminuyó en el 28 dpi por efecto de dicho tratamiento. Conclusiones. En condiciones experimentales, el aceite esencial de Aloysia triphylla tiene efecto anti-Trypanosoma cruzi in vivo en ratones.
\end{abstract}

Palabras clave: Trypanosoma cruzi; Cedrón; Aceites esenciales; Experimentación animal (fuente: DeCS BIREME).

\section{THE EFFECT OF THE ESSENTIAL OIL FROM Aloysia triphylla BRITTON (LEMON VERBENA) ON Trypanosoma cruzi IN MICE}

\begin{abstract}
Objectives. To determine the in-vivo anti-Trypanosoma cruzi activity of the essential oil from Aloysia triphylla in mice. Materials and methods. The mice $(n=15)$ in the study were randomly assigned to the following groups: infected and untreated (G1), infected and treated with benznidazole $100 \mathrm{mg} / \mathrm{kg}(\mathrm{G} 2)$, infected and treated with of Aloysia triphylla essential oil $100 \mathrm{mg} / \mathrm{kg}(\mathrm{G} 3)$, infected and treated with of Aloysia triphylla essential oil $250 \mathrm{mg} / \mathrm{kg}$ (G4), uninfected and untreated (G5), and uninfected and treated with $250 \mathrm{mg} / \mathrm{kg}$ of Aloysia triphyla (G6). The infection was conducted using 104 blood T. cruzi trypomastigotes and the treatment began on the 8th day post-infection (dpi) through 28 dpi. The parasitemia was determined through optical microscopy every 2 days using $5 \mu \mathrm{L}$ of blood sampled from the tail. In dpi 14 , 21 and 28, the blood sample was obtained from the queue for testing creatine kinase-MB, alanine aminotransferase and creatinine, then animals were euthanized and the heart was removed for histopathological study. Results. The essential oil of lemon verbena produced a significant reduction of the parasitemia, $85.4 \%$ with the peak at a the dose of $250 \mathrm{mg} /$ $\mathrm{kg}$. It was also observed a reduction in the number of amastigotes and inflammatory infiltrates in the heart. The creatine kinase-MB plasma levels also decreased at dpi 28 as a result of such treatment. Conclusions. Under experimental conditions, the essential oil of Aloysia triphylla (lemon verbena) has in vivo anti-Trypanosoma cruzi effect in mice.
\end{abstract}

Key words: Trypanosoma cruzi; Cedron; Oils, volatile; Animal experimentation (source: MeSH NLM).

\section{INTRODUCCIÓN}

La Aloysia triphylla Britton es una planta espontánea de América del Sur, originaria del Perú; pertenece a la familia de las Verbenáceas y también es conocida con el nombre botánico de Lippia citriodora Kunth, Lippia triphylla Kuntze, Aloysia citriodora Ortega, Verbena triphylla L'Héritier, Zapania citriodora Lam,
Aloysia sleumeri Mold, Aloysia triphylla (L'Herit.) Britt; Lippia citriodora H.B.K., Aloysia citriodora Ort. ex Pers.; mientras que, popularmente, se la conoce como "cedrón", "cidrón", "limón verbena", "verbena", "yerba luisa" o "hierba de la princesa", según el país o la región ${ }^{(1)}$. Es un arbusto perenne que puede medir más de $1,5 \mathrm{~m}$ de altura y se caracateriza porque sus hojas desprenden un intenso y agradable olor a limón;

\footnotetext{
Instituto de Investigaciones Clínicas, Facultad de Medicina Humana, Universidad Nacional Mayor de San Marcos, Lima, Perú.

2 Instituto de Medicina Tropical, Facultad de Medicina Humana, Universidad Nacional Mayor de San Marcos, Lima, Perú.

Químico farmacéutico, doctor en Farmacia y Bioquímica; ${ }^{b}$ médico, doctor en Medicina; ${ }^{c}$ médico patólogo
} 
su nombre "triphylla" se debe a que sus hojas simples, rugosas e insertadas en cada nudo, están reunidas en vértices de tres; sus flores son pequeñas, blancas por fuera y violáceas por dentro ${ }^{(2)}$.

La información etnofarmacológica refiere diversos usos de la $A$. triphylla en la medicina popular; la ingesta por vía oral de la infusión o decocción de las partes aéreas de esta planta se utiliza en Sudamérica como antiespasmódico, tranquilizante, calmante nervioso, expectorante y estomacal (1); además de este uso, en Bolivia se utiliza también para la hipertensión arterial (3); en Ecuador para tratar fiebre, dolor de cabeza y como diurético ${ }^{(4)}$. En Asia también se utiliza tradicionalmente para tratar espasmos gastrointestinales, resfrío común y como sedativo ${ }^{(5)}$.

Estudios científicos han demostrado importantes propiedades antimicrobianas de A. triphylla. El extracto acetónico de las partes aéreas evidenció significativa actividad contra Bacillus subtilis, Staphylococcus aureus, Escherichia coli, Klebsiella pneumoniae y Proteus vulgaris ${ }^{(6)}$; el extracto etanólico fue activo contra Listeria monocytogenes (7); por otra parte, el aceite esencial mostró actividad contra Staphylococcus aureus, Staphylococcus epidermidis, Bacillus cereus, Escherichia coli, Klebsiella pneumoniae, Proteus mirabilis, Enterobacter aerogenes, Klebsiella ozaenae, Enterococcus sp, Bacillus subtilis y Candida albicans ${ }^{\left({ }^{(8)}\right.}$ y produjo inhibición in vitro de la replicación de cuatro serotipos del virus del dengue ${ }^{(9)}$.

La composición química del aceite esencial es inconstante y depende del método de extracción, de su duración y la temperatura, del estado y la procedencia de la planta, y de las condiciones geobotánicas y agrícolas de su cultivo; los principales componentes son neral, geranial, limoneno, espatulenol, y variaciones intrínsecas en la cantidad y calidad del resto de terpenos como $\alpha$-tujeno, $\alpha$-pineno, camfeno, mirceno, p-cimeno, Y-terpineno, linalol, camferol, dihidrolinalool, citronelol, mentona, isoborneol, a-terpineol, carvona, etc (10). Según el componente principal se han identificado cinco quimiotipos: I, mircenona (37\%) y a-tujona (17\%); II, a-tujona (23\%) y cis-carveol (18\%), ambos en Argentina; III, 1,8-cineol (12\%) y geranial (10\%), en Marruecos; IV, limoneno (37\%), geranial (14\%) y neral (11\%), en Turquía; V, neral (10\%) y geranial $(40 \%)$, es el más abundante en el mundo y se encuentra principalmente en Colombia, Chile, Brasil, Eslovenia, Portugal, Francia y Grecia $^{(2)}$.

La trypanosomiasis americana o enfermedad de Chagas es una infección parasitaria causada por el protozoo Trypanosoma cruzi, transmitida por insectos hematófagos de la familia Reduviidae. Esta enfermedad está presente en casi todos los países de Latinoamérica con una prevalencia global de ocho a nueve millones de personas y una población en riesgo de 25 millones (11). En los últimos años, la rapidez de los viajes en avión y las migraciones, han llevado a la exportación de casos de enfermedad de Chagas fuera de Latinoamérica a países como Alemania, Australia, Canadá, España, Francia, Italia, Japón o EE. UU. (12).

La farmacoterapia actual de la enfermedad de Chagas comprende escasamente dos fármacos, nifurtimox y benznidazol; ambos son tóxicos, es necesario administrarlos por largo tiempo y solo sirven para la fase aguda de la enfermedad (13). Ante esta situación, es urgente la búsqueda de nuevos agentes terapéuticos donde las plantas medicinales son una opción viable; no obstante, la inmensa mayoría de estudios de la actividad tripanocida con derivados de plantas, se han realizado solamente por medio de ensayos in vitro. En un estudio recientemente publicado, hemos demostrado que el aceite esencial de $A$. triphylla inhibió el crecimiento in vitro de epimastigotes de $T$. cruzi con una moderada $\mathrm{Cl}_{50}$ de $96,5 \mu \mathrm{g} / \mathrm{mL}{ }^{(14)}$. Esto nos motivó a continuar el estudio, por lo que nos propusimos evaluar in vivo el efecto anti-Trypanosoma cruzi del aceite esencial de $A$. triphylla en un modelo de ratón.

\section{MATERIAL Y MÉTODOS}

Se realizó un estudio experimental en ratones albinos machos $(22 \pm 2 \mathrm{~g})$ obtenidos del Instituto Nacional de Salud de Perú y mantenidos bajo un ciclo de luz/oscuridad de 12/12 horas, con un periodo de aclimatación de una semana antes del experimento, recibiendo alimento y agua ad libitum.

\section{PREPARACIÓN DEL ACEITE DE Aloysia triphylla}

Se recolectó muestras de la Aloysia triphylla en la ciudad de Huancayo, Perú; estas muestras fueron llevadas al Museo de Historia Natural de la Universidad Nacional Mayor de San Marcos para su identificación taxonómica.

El aceite esencial se obtuvo de las hojas frescas, a través de una destilación por arrastre con vapor de agua en un aparato tipo clevenger; el destilado se separó tomando en cuenta sus propiedades de inmiscibilidad y diferencia de densidades entre el agua y el aceite esencial, utilizando una pera de separación de vidrio. Se deshidrató con sulfato sódico anhidro $\left(\mathrm{Na}_{2} \mathrm{SO}_{4}\right)$, se filtró y se conservó en un frasco de vidrio color ámbar bajo refrigeración a una temperatura de $4^{\circ} \mathrm{C}$ hasta su uso. 


\section{OBTENCIÓN DEL Trypanosoma cruzi}

Se utilizó una cepa silvestre no estandarizada de Trypanosoma cruzi aislada del vector Triatoma infestans colectado en el departamento de Arequipa, Perú. Los parásitos fueron mantenidos por pases de sangre infectada a ratones albinos Balb/c normales. Se realizaron dos pases previos por ratón antes de la realización del experimento.

En el ensayo se usaron tripomastigotes sanguíneos cosechados por punción cardíaca de los ratones infectados por Trypanosma cruzi, en el pico de parasitemia.

\section{GRUPOS EXPERIMENTALES}

Los animales fueron asignados aleatoriamente a los siguientes grupos ( 15 ratones por grupo): infectados y no tratados (G1); infectados y tratados con benznidazol 100 $\mathrm{mg} / \mathrm{kg}(\mathrm{G} 2)$; infectados y tratados con aceite esencial de Aloysia triphylla $100 \mathrm{mg} / \mathrm{kg}$ (G3); infectados y tratados con aceite esencial de Aloysia triphylla $250 \mathrm{mg} / \mathrm{kg}$ (G4); no infectados y no tratados (G5) y no infectados y tratados con $250 \mathrm{mg}$ de Aloysia triphylla (G6).

La infección se realizó por inyección intraperitoneal de $10^{4}$ tripomastigotes sanguíneos.

El aceite esencial fue disuelto en dimetilsulfóxido (DMSO) al 1\% y se administró una vez al día mediante una sonda orogástrica en las dosis indicadas a los grupos G3, G4 y G6, desde el 8 al 28 dpi (día postinfección).

El benznidazol fue administrado en dosis de $100 \mathrm{mg} /$ $\mathrm{kg} /$ día por vía oral (grupo G2); mientras que los grupos G1 y G5 solamente recibieron el vehículo utilizado en la muestra. El inicio del tratamiento fue desde el día 8, en un esquema similar a los grupos anteriores.

\section{CONTROL DE LA EXPERIMENTACIÓN}

La infección se determinó desde el día 4 y la experimentación comenzó el día 8 con el inicio del tratamiento. En los ratones se evaluó el nivel de parasitemia, peso corporal, isoenzima cardíaca creatina kinasa (CK-MB), alanina aminotransferasa (ALT) y creatinina.

Medición de la parasitemia: fue determinada individualmente cada dos días por microscopía directa contando los parásitos en $5 \mu \mathrm{L}$ de sangre obtenida de la cola de los ratones. Se observó al microscopio a 400X y se realizó el recuento de tripomastigotes en cincuenta campos. La parasitemia se calculó multiplicando el número de parásitos en cincuenta campos por el factor de microscopio.
Medición del CK-MB, ALT y creatinina: se colectó sangre de la punta de la cola de los ratones de todos los grupos experimentales en microcapilares heparinizados, en los días 14, 21 y 28 postinfección. El plasma fue obtenido después de la centrifugación de la sangre en una centrífuga de microhematocrito. Se determinó los niveles plasmáticos de la CK-MB, ALT y creatinina.

Se determinó la actividad de la CK-MB usando un slide CKMB (Vitros $\left.{ }^{\circledR}\right)$. Se depositó en el slide, una gota de $11 \mu \mathrm{L}$ de plasma que fue distribuida uniformemente por la capa difusora a las capas subyacentes. En la capa reactiva, la creatina quinasa en la muestra cataliza la conversión del fosfato de creatina y del difosfato de adenosina (ADP) a creatina y trifosfato de adenosina (ATP). En presencia de glicerol quinasa, el glicerol es fosforilado a L- $\alpha$-glicerofosfato oxidasa. Finalmente, un leucocolorante es oxidado por el peróxido de hidrógeno en presencia de peroxidasa para formar un colorante. El grado de variación de la densidad de reflexión se convierte en actividad enzimática. La lectura se realizó a 670 nm.

Para la medición de la ALT y creatinina se utilizó slides ALT y CREA (Vitros $®$ ). Los resultados de ALT fueron expresados como concentración de enzima (UI/L). Los niveles de creatinina se expresaron como $\mathrm{mg} / \mathrm{dL}$.

\section{EVALUACIÓN HISTOPATOLÓGICA}

En el 14, 21 y $28 \mathrm{dpi}$, se seleccionaron aleatoriamente cinco ratones de cada grupo que fueron sacrificados por dislocación cervical y los corazones fueron rápidamente extraídos y procesados para análisis histopatológico. Los corazones se cortaron longitudinalmente, se lavaron en tampón fosfato salino (PBS) helado y se fijaron en formaldehído al $10 \%$ en PBS. Los tejidos fijados fueron deshidratados y embebidos en parafina. Se realizaron secciones de $3 \mu \mathrm{m}$, que fueron coloreadas con hematoxilina-eosina (HE) y se analizaron por microscopía de luz.

Se determinó el número de nidos de amastigotes y de infiltrados inflamatorios (más de diez células mononucleares) en cien campos en cada lámina, a 400X. Se obtuvo el número promedio de nidos de amastigotes o infiltrados inflamatorios por campo, con tres secciones por cada muestra de cada ratón.

\section{ANÁLISIS DE DATOS}

Los datos obtenidos fueron expresados como media \pm error estándar y las comparaciones entre los grupos experimentales fueron realizadas por ANOVA de una vía seguido por una prueba post-hoc de Scheffé. Las medias se consideraron significativamente diferentes cuando $p<0,05$. Se utilizó el programa estadístico SPSS 15.0 . 


\section{RESULTADOS}

En los primeros 8 dpi se verificó la infección con Trypanosoma cruzi en el $100 \%$ de los ratones inoculados y no se observó diferencias significativas en el nivel de parasitemia en los grupos infectados.

\section{INFECCIÓN POR Trypanosoma cruzi}

El tratamiento a partir del $8 .^{\circ} \mathrm{dpi}$ con el aceite esencial de Aloysia triphylla (cedrón) en dosis de $250 \mathrm{mg} / \mathrm{kg} / \mathrm{día} \mathrm{por}$ vía oral produjo una reducción significativa de $85,4 \%$ $(p<0,05)$ en el pico de la parasitemia en el $22 \mathrm{dpi}$. Con la dosis de cedrón de $100 \mathrm{mg} / \mathrm{kg} /$ día se produjo una menor reducción de la parasitemia $(41,8 \%)$. La parasitemia se redujo $100 \%$ con el tratamiento con $100 \mathrm{mg} / \mathrm{kg} / \mathrm{día}$ de Benznidazol (Figura 1).

El mayor número de nidos de amastigotes en tejido cardiaco se observó en el 28 dpi en el grupo infectado y no tratado $(46,4 \pm 10,7$ nidos de amastigotes/100

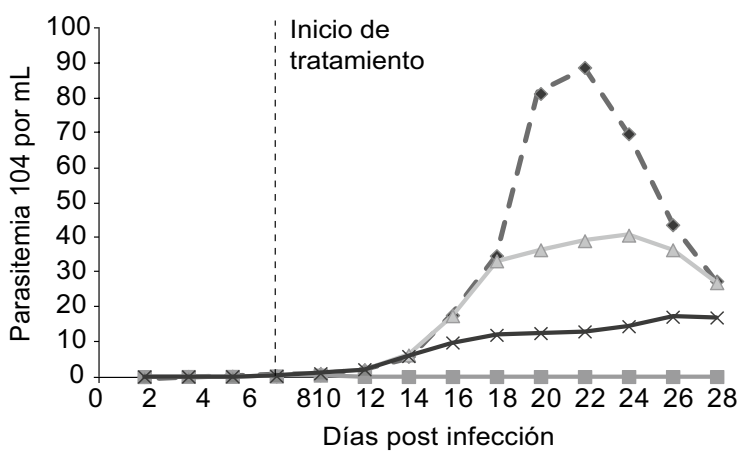

- No tratado - - Benznidazol $\triangle$ - Cedrón $100 \multimap$ Cedrón 250

Figura 1. Efecto del tratamiento por vía oral con aceite esencial de cedrón sobre la parasitemia en ratones infectados con $10^{4}$ tripomastigotes sanguíneos de $T$. cruzi durante 28 días.

campos), seguido por el grupo infectado y tratado con $100 \mathrm{mg} / \mathrm{kg}$ de cedrón $(16,8 \pm 12,3$ nidos de amastigotes $/ 100$ campos) produciendo dicho tratamiento una reducción de $63,8 \%$. En los grupos tratados con benznidazol $100 \mathrm{mg} / \mathrm{kg}$ y cedrón $250 \mathrm{mg} / \mathrm{kg}$ no se observaron nidos de amastigotes en el 28 dpi (Figuras 2, 3 y 4).

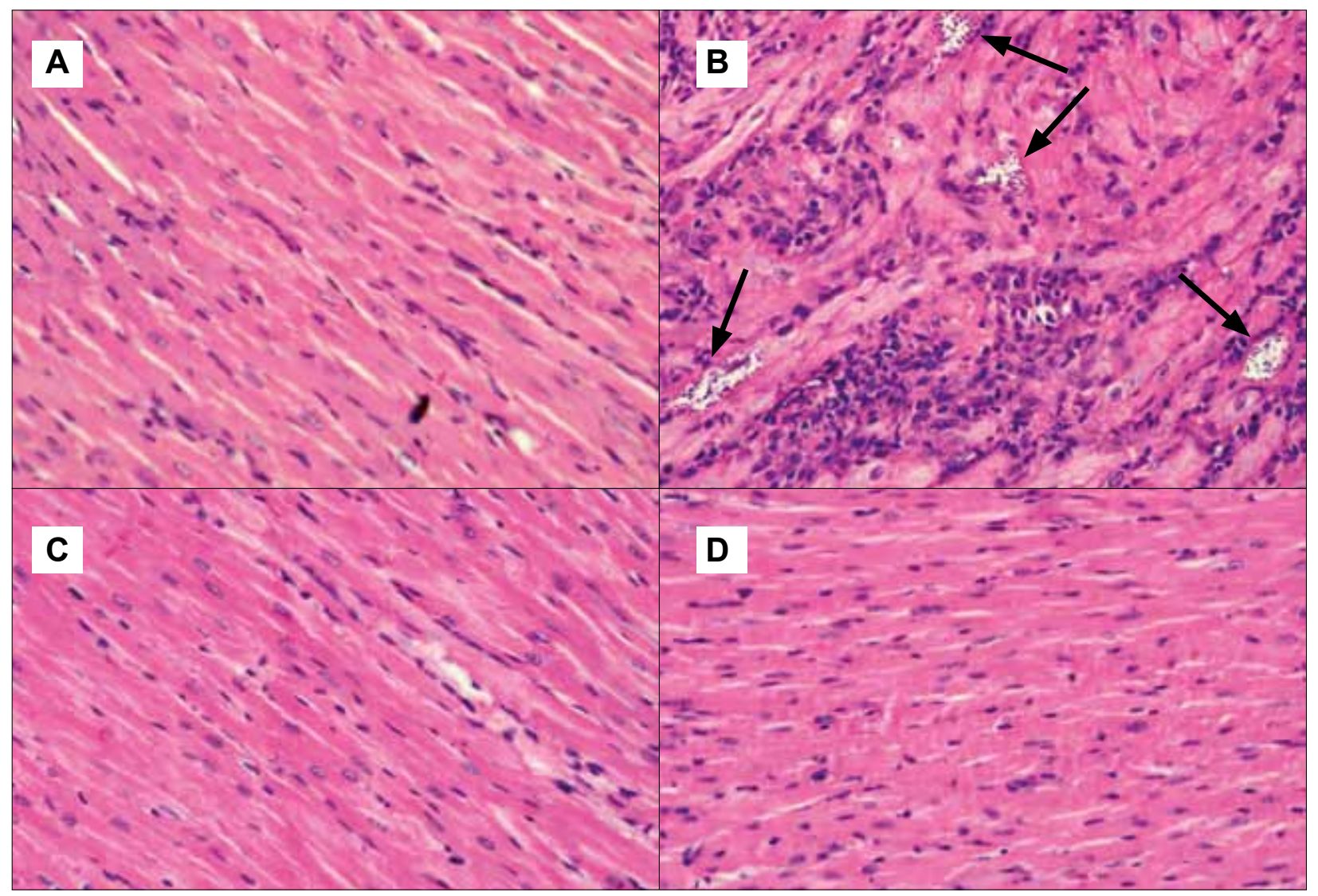

Figura 2. Vista panorámica de tejido cardiaco de ratones BALB/c infectados con Trypanosoma cruzi, en el 28 dpi, después del tratamiento con el aceite esencial de Aloysia triphylla y benznidazol a partir del 8 dpi. (Coloración HE 400X).

(A) Tejido cardiaco normal (control) en el grupo no infectado con T. cruzi. (B) nidos de amastigotes (Flechas) e infiltrados inflamatorios en el grupo infectado con T. cruzi y sin tratamiento con fármacos. (C) Grupo infectado con T. cruzi y tratado con benznidazol $100 \mathrm{mg} /$ $\mathrm{kg} / \mathrm{día}$. (D) Grupo infectado con T. cruzi y tratado con aceite esencial de Aloysia triphylla $250 \mathrm{mg} / \mathrm{kg} / \mathrm{día}$. 


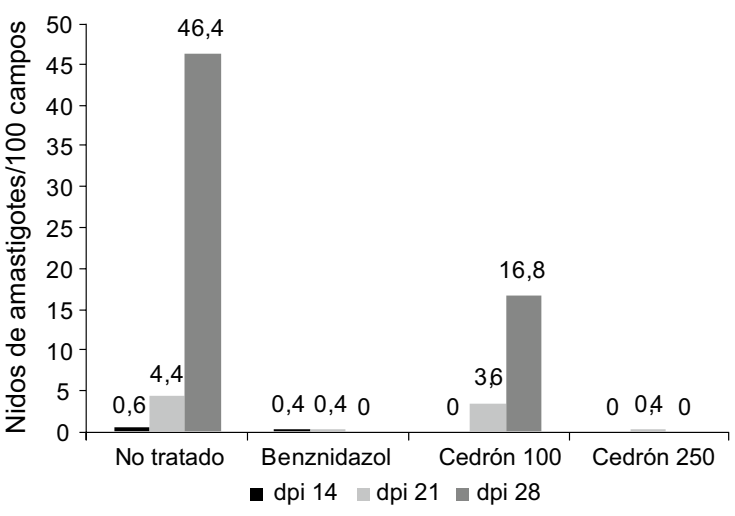

Figura 3. Nidos de amastigotes/100 campos observados a 400X en corazón de ratones infectados con $10^{4}$ tripomastigotes sanguíneos de $T$. cruzi y tratados con benznidazol y cedrón.

El número de infiltrados inflamatorios/100 campos se incrementó progresivamente en el grupo infectado y no tratado, siendo mayor en el $28 \mathrm{dpi}$. El tratamiento con benznidazol y cedrón $250 \mathrm{mg} / \mathrm{kg}$ produjo una reducción significativa de infiltrados inflamatorios en el 28 dpi $(p<0,001)$, mientras que con $100 \mathrm{mg} / \mathrm{kg}$ de cedrón, la reducción fue menor pero significativa $(p<0,01)$.

\section{ALTERACIÓN EN ANÁLISIS BIOQUÍMICOS Y PESO CORPORAL}

La ganacia de peso corporal fue alterada en los ratones infectados, produciéndose una disminución significativa de peso $(p<0,05)$ en el $21 \mathrm{dpi}$, que se incrementó en el 28 dpi en todos los grupos, siendo mayor en los grupos que recibieron tratamiento con cedrón en dosis de 250 $\mathrm{mg} / \mathrm{kg} / \mathrm{día}$, sea infectado o no $(p<0,01)$, excepto en el grupo que recibió tratamiento con benznidazol, donde si hubo ganancia de peso que, aunque fue menor, la diferencia no fue significativa (Figura 4).

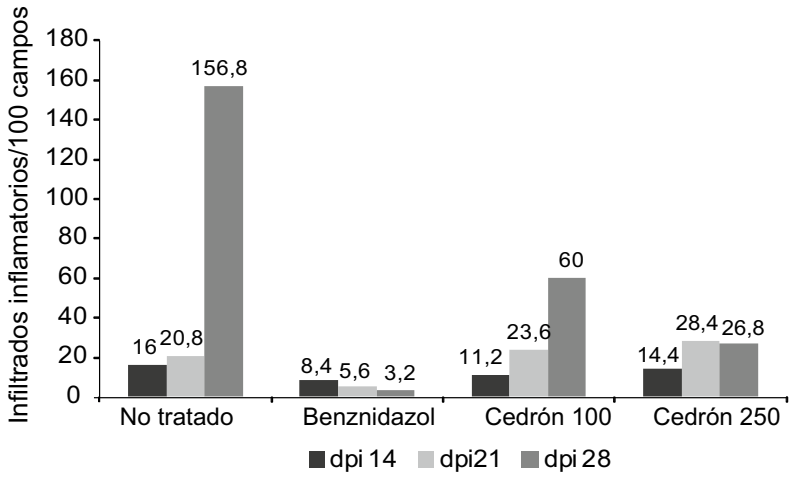

Figura 4. Infiltrados inflamatorios/100 campos observados a 400X en corazón de ratones infectados con $10^{4}$ tripomastigotes sanguíneos de $T$. cruzi y tratados con benznidazol y cedrón.

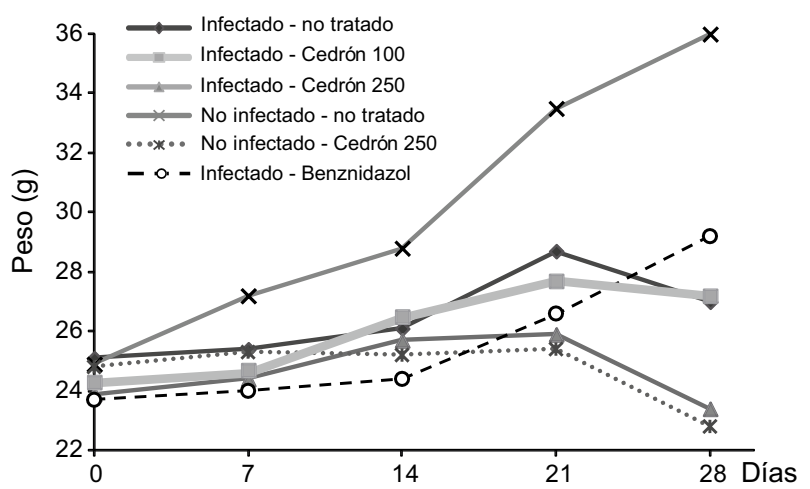

Figura 5. Variación del peso corporal de ratones infectados con $10^{4}$ tripomastigotes de T. cruzi tratados con benznidazol y cedrón comparado con ratones no infectados y no tratados.

Los niveles plasmáticos de CK-MB se incrementaron significativamente desde el $21 \mathrm{dpi}$ en los grupos infectados $(p<0,001)$, excepto en el grupo tratado con benznidazol. El incremento continuó hasta el 28 dpi en los grupos G1 y G2 (infectado-no tratado e infectadotratado con $100 \mathrm{mg} / \mathrm{kg}$ de cedrón, respectivamente), mientras que en el grupo infectado y tratado con cedrón $250 \mathrm{mg} / \mathrm{kg}$ descendió en el intervalo del 21 al 28 dpi, pero se mantiene más elevado respecto a los controles (no infectados y tratados con benznidazol), que se muestran en la Tabla 1.

Los niveles plasmáticos de ALT se incrementaron significativamente en todos los grupos infectados desde el 21 dpi inclusive en el grupo infectado y tratado con benznidazol. Los niveles de dicha enzima continuaron incrementados significativamente $(p<0,001)$ en el $28 \mathrm{dpi}$.

Los niveles plasmáticos de creatinina se muestran en la Tabla 1. Los valores se incrementaron significativamente desde los $21 \mathrm{dpi}$ y disminuyeron levemente en el $28 \mathrm{dpi}$.

\section{DISCUSIÓN}

En esta investigación se demuestra que el pico de parasitemia al utilizar una cepa peruana de Trypanosoma cruzi se produjo en la tercera semana postinfección (Figura 1). En otros estudios que han utilizado cepas procedentes de otros países latinoamericanos se ha demostrado que el pico de parasitemia se alcanzó en diferentes tiempos; así, tenemos que para el caso de la cepa Tulahuen de Chile y cepa $Y$ de Brasil, el pico de parasitemia se produjo en la primera semana ${ }^{(15,16)}$, mientras que para las cepas $\mathrm{X}-1$ y $\mathrm{K}-1$ de Argentina se produjo en el 18 y 22 dpi, respectivamente ${ }^{(17)}$. Este particular comportamiento de las cepas de T. cruzi ha sido asociado con su origen geográfico; al respecto, $T$. cruzi presenta un alto grado de variabilidad genética intraespecífica, con posible implicación para la forma 
Tabla 1. Niveles plasmáticos de ALT (UI/L), CK-MB (U/L) y creatinina $(\mathrm{mg} / \mathrm{dL})$ en ratones infectados con $10^{4}$ tripomastigotes sanguíneos de $T$. cruzi y tratados con benznidazol y cedrón.

\begin{tabular}{|c|c|c|c|c|c|c|}
\hline & $\begin{array}{l}\text { No infectado- } \\
\text { No tratado }\end{array}$ & $\begin{array}{c}\text { Infectado- } \\
\text { Benznidazol } \\
100 \mathrm{mg} / \mathrm{kg} / \mathrm{día}\end{array}$ & $\begin{array}{l}\text { Infectado- } \\
\text { Cedrón } 100 \\
\text { mg/kg/día }\end{array}$ & $\begin{array}{l}\text { Infectado-Cedrón } \\
250 \text { mg/kg/día }\end{array}$ & $\begin{array}{l}\text { Infectado- } \\
\text { No tratado }\end{array}$ & $\begin{array}{c}\text { No infectado- } \\
\text { Cedrón } 250 \text { mg/ } \\
\text { kg/día }\end{array}$ \\
\hline \multicolumn{7}{|l|}{$A L T$} \\
\hline 14 dpi & $21,8 \pm 1,66$ & $21,6 \pm 1,96$ & $23,2 \pm 1,43$ & $21,2 \pm 1,93$ & $22,2 \pm 2,65$ & $21,2 \pm 1,77$ \\
\hline $21 \mathrm{dpi}$ & $21,4 \pm 1,36$ & $62,4 \pm 3,26^{*}$ & $58,4 \pm 3,17^{*}$ & $47,4 \pm 2,54^{*}$ & $61,4 \pm 2,31^{*}$ & $41,6 \pm 2,66^{* *}$ \\
\hline $28 \mathrm{dpi}$ & $22,2 \pm 1,65$ & $59,8 \pm 3,73^{*}$ & $55,0 \pm 2,17^{*}$ & $52,2 \pm 4,09^{*}$ & $65,0 \pm 4,18^{*}$ & $52,8 \pm 3,02^{*}$ \\
\hline \multicolumn{7}{|l|}{ CK-MB } \\
\hline $14 \mathrm{dpi}$ & $38,6 \pm 2,79$ & $51,0 \pm 8,25$ & $45,4 \pm 6,70$ & $48,0 \pm 1,14$ & $62,4 \pm 5,01$ & ND \\
\hline $21 \mathrm{dpi}$ & $40,0 \pm 2,45$ & $52,0 \pm 4,11$ & $138,8 \pm 5,40^{*}$ & $130,8 \pm 3,12^{*}$ & $150,8 \pm 4,11^{*}$ & ND \\
\hline $28 \mathrm{dpi}$ & $39,2 \pm 1,39$ & $49,4 \pm 2,44$ & $278,0 \pm 12,9^{*}$ & $112,6 \pm 5,59^{*}$ & $381,20 \pm 9,6^{*}$ & ND \\
\hline \multicolumn{7}{|c|}{ CREATININA } \\
\hline 14 dpi & $0,69 \pm 0,22$ & $0,77 \pm 0,07$ & $0,99 \pm 0,09$ & $0,89 \pm 0,06$ & $0,85 \pm 0,13$ & $0,69 \pm 0,13$ \\
\hline $21 \mathrm{dpi}$ & $0,73 \pm 0,06$ & $1,97 \pm 0,07^{*}$ & $1,35 \pm 0,15$ & $1,57 \pm 0,24^{\star * *}$ & $1,87 \pm 0,18^{* *}$ & $1,52 \pm 0,05^{\star * *}$ \\
\hline $28 \mathrm{dpi}$ & $0,71 \pm 0,05$ & $1,25 \pm 0,18$ & $1,18 \pm 0,20$ & $1,39 \pm 0,19$ & $1,56 \pm 0,15^{\text {***}}$ & $1,33 \pm 0,06$ \\
\hline
\end{tabular}

Valores expresados como media aritmética \pm error estándar.

${ }^{*} p<0,001$ frente a grupo no infectado, ${ }^{* *} p<0,01$ frente a grupo no infectado, ${ }^{* * *} p<0,05$ frente a grupo no infectado.

ND: no determinado.

clínica de la enfermedad, como el desarrollo de cardiopatía, megaesófago y megacolon, solos o en combinación ${ }^{(18)}$.

En la búsqueda de nuevas drogas alternativas para el tratamiento de la enfermedad de Chagas, los extractos vegetales y los aceites esenciales aparecen como prometedores agentes antiproliferativos. El extracto diclorometano de la hoja de Baccharis dracunculifolia, a una concentración de $3 \mathrm{mg} / \mathrm{mL}$, mostró $100 \%$ de lisis de formas tripomastigotes de T. cruzi ${ }^{(19)}$. Los extractos y fracciones de Ampelozizyphus amazonicus exhibieron lisis de las formas tripomastigotes de T. cruzi in vitro ${ }^{(20)}$. Por otra parte, los aceites esenciales también han demostrado importante actividad contra T. cruzi. Se ha descrito en estudios in vitro que el aceite esencial de Origanum vulgare (orégano) inhibió el crecimiento de epimastigotes de T. cruzi y también indujo lisis de tripomastigotes, semejante actividad se observó con el aceite esencial de Thymus vulgaris $\mathrm{L}$ (tomillo) ${ }^{(21)}$. Los aceites esenciales de Achillea millefolium $L$ (milenrama), Syzygium aromaticum $\mathrm{L}$ (clavo) y Ocimun basilicum L (albahaca) también han demostrado su actividad inhibitoria del desarrollo de epimastigotes y tripomastigotes de T. cruzi (22). Recientemente se ha informado la eficacia in vitro del extracto etanólico de hojas de Zanthoxylum chiloperone y de la canthin-6-ona, principal componente de la corteza del tallo y la raíz, contra las formas tripomastigotes y amastigotes de $T$. cruzi, así como la eficacia del extracto etanólico de las hojas en la reducción de la parasitemia por T. cruzi en ratones Balb/c ${ }^{(23)}$. Por otra parte, el derivado fenólico de un alcaloide naftilisoquinolínico aislado de Ancistrocladus cochinchinensis mostró excelente actividad contra T. cruzi ${ }^{(24)}$.
En la presente investigación, el aceite esencial de Aloysia triphylla (cedrón) en dosis de $250 \mathrm{mg} / \mathrm{kg}$ produjo una reducción significativa del pico de parasitemia de $85,4 \%$ en el 22 dpi (Figura 1), esto guarda relación con la reducción significativa del número de nidos de amastigotes e infiltrados inflamatorios que se observa en el tejido cardiaco al final del experimento (Figuras 2 y 3). Este efecto podría ser importante ya que si bien no produjo la eliminación de los parásitos de manera similar a benznidazol, es generalmente aceptado que con una infección aguda más severa se puede desarrollar una fase crónica más agresiva ${ }^{(25)}$. Por otra parte, los niveles plasmáticos de CK-MB se incrementaron significativamente en la tercera semana en los grupos infectados con Trypanosoma cruzi, excepto el grupo tratado con benznidazol; al respecto, se ha demostrado que la infección por $T$. cruzi produce incremento plasmático de CK-MB comparado con ratones control no infectados, que revelaría indirectamente la invasión del músculo cardiaco por este parásito protozoario (17); por su parte, de Souza et al., observaron que después de tres semanas, los ratones infectados con $10^{4}$ parásitos mostraron incremento en los niveles plasmáticos de CK-MB; asimismo, encontraron una correlación positiva entre los infiltrados inflamatorios en tejido cardiaco detectado histopatológicamente en los animales y los niveles de $\mathrm{CK}-\mathrm{MB}$, indicando que este marcador puede ser útil para monitorear la ocurrencia de miocarditis chagásica experimental ${ }^{(26)}$. En este estudio, los niveles plasmáticos de CK-MB disminuyeron significativamente $(p<0,001)$ en el 28 dpi por efecto del tratamiento con aceite esencial de cedrón en dosis de $250 \mathrm{mg} / \mathrm{kg}$ (Tabla 1), lo que también guarda relación con la nula observación de amastigotes en el tejido cardiaco de los 
ratones de este grupo al final del experimento (Figura 2) y los escasos infiltrados inflamatorios.

En el grupo no tratado e infectado con Trypanosoma cruzi, se observó un incremento progresivo del número de infiltrados inflamatorios. Durante la infección con T. cruzi, la activación del sistema inmune innato está probablemente involucrado en, al menos, dos aspectos de la fisiopatología de la enfermedad de Chagas, el control de la replicación y dispersión del parásito en el tejido del huésped y la reacción inflamatoria en el tejido infectado del huésped, siendo considerado este último como la causa principal de daño tisular que conduce a disfunción del órgano durante la fase aguda y crónica de la enfermedad (27). La miocarditis aguda por T. cruzi está asociada con nidos de parásitos y focos inflamatorios celulares que están compuestos de macrófagos, células $\mathrm{CD}^{+}$y principalmente $\mathrm{CD} 8^{+}$, diferenciadas como células T activadas/memoria (CD62 Low, LFA-1 ${ }^{\text {High }}$ y VLA-4 ${ }^{\text {High }}{ }^{(28)}$. Se ha demostrado la presencia de moléculas ancladas de glicosilfosfatidilinositol (GPI) en la membrana plasmática de $T$. cruzi que son los antígenos predominantes que actúan sobre el receptor TLR2 provocando la síntesis de citocinas proinflamatorias y quimiocinas en macrófagos ${ }^{(27) .}$

El incremento de los niveles plasmáticos de ALT y creatinina en ratones infectados con $T$. cruzi (Tabla 1) concuerda con los hallazgos de De Souza et al., (16) quienes demostraron que la infección por $T$. cruzi induce importantes lesiones hepáticas y renales. También se ha informado que la cepa Tulahuen de $T$. cruzi indujo serias lesiones hepáticas caracterizadas por la desorganización de la estructura trabecular, infiltración de células mononucleares y la presencia de amastigotes en el interior de ellas ${ }^{(17)}$. Además, se ha demostrado que ratones Balb/c infectados con $T$. cruzi, en el $15 \mathrm{dpi}$ presentaron atrofia glomerular y aparente necrosis, con discreta hemorragia glomerular y degeneración celular cerca de los túbulos proximales, con lo que los ratones desarrollaron un síndrome cardio/anémico/ renal en la fase aguda de la infección que se acompañó de un incremento en los niveles de creatinina (29). Se ha demostrado también que previo a la alteración cardiaca, los ratones infectados presentan infiltración inflamatoria renal que causa daño renal agudo debido a una lesión por isquemia/reperfusión provocado por citocinas proinflamatorias y altas concentraciones de óxido nítrico que, si bien lo protege de la invasión del parásito, causa daño de la integridad y viabilidad celular renal ${ }^{(25)}$. Existe la posibilidad que esto esté relacionado con la significativa pérdida de peso corporal que se observó en los grupos (Figura 4) y que fue mayor en los grupos tratados con aceite esencial de cedrón en dosis de $250 \mathrm{mg} / \mathrm{kg}$, pero al observarse similar efecto en el grupo no infectado y tratado con la misma dosis de cedrón, se podría especular que el aceite de cedrón podría cumplir algún papel en la toxicidad, lo cual podría estar relacionada con la alta dosis utilizada, ya que en el ensayo con células murinas no mostró citotoxicidad (14), por lo que sería necesario realizar estudios de toxicidad aguda y crónica para establecer la seguridad de su potencial uso como agente anti-Trypanosoma cruzi.

Es probable que la actividad del aceite esencial de Aloysia triphylla contra $T$. cruzi podría deberse a su principal componente, el citral, una mezcla de dos aldehídos monoterpénicos isoméricos, geranial y neral; el isómero trans- se conoce como geranial o citral $A$, y el isómero cis- se conoce como neral o citral $\mathrm{B}^{(2)}$. Esto sería posible porque se ha demostrado in vitro que el citral en concentración mayor de $60 \mu \mathrm{g} / \mathrm{mL}$ produce $100 \%$ de lisis de formas epimastigotes y tripomastigotes de $T$. cruzi ${ }^{(30)}$.

En conclusión, en las condiciones experimentales del estudio, el aceite esencial de Aloysia triphylla Britton (cedrón) tiene efecto anti-Trypanosoma cruzi in vivo en ratones.

\section{Contribuciones de autoría}

JR, OP y SR participaron de la concepción y diseño del estudio, en la recolección de resultados, en el análisis e interpretación de datos, redacción del manuscrito, revisión crítica del artículo y la aprobación de la versión final a publicar. JR contribuyó en la obtención del financiamiento y recolección de datos, OP en el estudio parasitológico, y GR en los análisis bioquímicos.

\section{Fuentes de financiamiento}

El estudio fue financiado por el Vicerrectorado de Investigación de la Universidad Nacional Mayor de San Marcos.

\section{Conflictos de interés}

Los autores declaran no tener conflictos de interés en la ejecución y publicación de este artículo.

\section{REFERENCIAS BIBLIOGRÁFICAS}

1. Gupta M. 270 Plantas medicinales Iberoamericanas. $1^{\mathrm{a}}$ ed. Santa Fe de Bogotá: Editorial Presencia Ltda.; 1995.

2. Díaz O. Estudio comparativo de la composición química y evaluación de la actividad antioxidante del aceite esencial de Aloysia triphylla (L' Her) Britton, cultivada en tres regiones de Colombia. Tesis para optar el título de Químico. Bucaramanga, Colombia: Universidad Industrial de Santander; 2007.

3. Macía MJ, García E, Vidaurre PJ. An ethnobotanical survey of medicinal plants commercialized in the markets of $\mathrm{LaPaz}$ and El Alto, Bolivia. J Ethnopharmacol. 2005;97(2):337-50.

4. Tene V, Malagón O, Vita PV, Vidari G, Armijos C, Zaragoza T. An ethnobotanical survey of medicinal plants used in Loja and Zamora-Chinchipe, Ecuador. J Ethnopharmacol. 2007;111(1):63-81. 
5. Mothana RA, Abdo SA, Hasson S, Althawab FM, Alaghbari SA, Lindequist U. Antimicrobial, antioxidant and cytotoxic activities and phytochemical screening of some yemeni medicinal plants. Evid Based Complement Alternat Med. 2010;7(3):323-30.

6. Kumar NK, Kumar KS, Raman B, Reddy I, Ramarao M, Rajagopal S. Antibacterial activity of Lippia citriodora a folklore plant. JPAM. 2008;2(1):249-52.

7. Bayoub K, Baibai T, Mountassif D, Retmane A, Soukri A. Antibacterial activities of the crude ethanol extracts of medicinal plants against Listeria monocytogenes and some other pathogenic strains. Afr J Biotechnol. 2010;9(27):4251-8.

8. Oliva M, Beltramino E, Gallucci M, Casero C, Zygadlo J, Demo M. Antimicrobial activity of essential oils of Aloysia triphylla (L'Her.) Britton from different regions of Argentina. BLACPMA. 2010;9(1):29-37.

9. Ocazionez RE, Meneses R, Torres FA, Stashenko E. Virucidal activity of Colombian Lippia essential oils on dengue virus replication in vitro. Mem Inst Oswaldo Cruz. 2010;105(3):304-9.

10. Di Leo Lira $P$, van Baren CM, Retta D, Bandoni AL, Gil $\mathrm{A}$, Gattuso $\mathrm{M}$, et al. Characterization of lemon verbena (Aloysia citriodora Palau) from Argentina by the essential oil. J Essent Oil Res. 2008;20(4):350-3.

11. Hotez PJ, Molyneux DH, Fenwick A, Kumaresan J, Sachs SE, Sachs JD, et al. Control of neglected tropical diseases. N Engl J Med 2007;357(10):1018-27.

12. Organización Mundial de la Salud. Enfermedad de Chagas: 100 años después. Boletín de la Organización Mundial de la Salud [Internet]. 2009 [citado el 5 diciembre de 2011];87:485-564. Disponible en: http://www.who.int/ bulletin/volumes/87/7/09-030709/es/

13. Brunton LL, Lazo JS, Parker KL. Goodman \& Gilman. Las bases farmacológicas de la terapéutica. $11^{\mathrm{a}}$ ed. México, DF: McGraw-Hill Interamericana; 2007.

14. Rojas J, Solís H, Palacios O. Actividad anti-Trypanosoma cruzi in vitro de aceites esenciales de diez plantas medicinales. An Fac Med. 2010;71(3):161-5.

15. Saraiva J, Vega C, Rolon M, da Silva R, E Silva ML, Donate $\mathrm{PM}$, et al. In vitro and in vivo activity of lignan lactones derivatives against Trypanosoma cruzi. Parasitol Res. 2007;100(4):791-5.

16. de Souza EM, Oliveira GM, Boykin DW, Kumar A, Hu Q, De Nazaré C, et al. Trypanocidal activity of the phenilsubstituted analogue of furamidine DB569 agaisnt Trypanosoma cruzi infection in vivo. J Antimicrob Chemother. 2006;58 (3):610-4.

17. Cano RC, Hliba E, Rubiolo ER. Creatine kinase and lactate dehydrogenase levels as potential indicators of Trypanosoma cruzi infectivity and histotropism in experimental Chagas' disease. Parasitol Res. 2000;86(3):244-52.

18. Manoel-Caetano Fda S, Silva AE. Implications of genetic variability of Trypanosoma cruzi for the pathogenesis of Chagas disease. Cad Saude Publica. 2007;23(10):2263-74.

19. da Silva Filho AA, Pires Bueno PC, Gregório LE, Andrade e Silva ML, Albuquerque S, Bastos JK. In-vitro trypanocidal activity evaluation of crude extract and isolated compounds from Baccharis dracunculifolia D.C. (Asteraceae). J Pharm Pharmacol. 2004;56(9):1195-9.
20. Rosas LV, Cordeiro MS, Campos FR, Nascimento SK, Januário $\mathrm{AH}$, França $\mathrm{SC}$, et al. In vitro evaluation of the cytotoxic and trypanocidal activities of Ampelozizyphus amazonicus (Rhamnaceae). Braz J Med Biol Res. 2007;40(5):663-70.

21. Santoro GF, das Graças Cardoso M, Guimarães LG, Salgado AP, Menna-Barreto RF, Soares MJ. Effect of orégano (Origanum vulgare L.) and Thyme (Thymus vulgaris L.) essential oils on Trypanosoma cruzi (Protozoa: Kinetoplastida) growth and ultraestructure. Parasitol Res. 2007;100(4):783-90.

22. Santoro GF, Cardoso MG, Guimarães LG, Mendonça LZ, Soares MJ. Trypanosoma cruzi: activity of essential oils from Achillea millefolium L., Syzygium aromaticum L. and Ocimun basilicum L. on epimastigotes and trypomastigotes. Exp Parasitol. 2007;116(3):283-90.

23. Ferreira ME, Cebrián-Torrejón G, Corrales AS, Vera de Bilbao N, Rolón M, Gomez CV, et al. Zanthoxylum chiloperone leaves extract: first sustainable Chagas disease treatment. J Ethnopharmacol. 2011;133(3):986-93.

24. Bringmann G, Hertlein-Amslinger B, Kajahn I, Dreyer M, Brun R, Moll H, et al. Phenolic analogs of the N,C-coupled naphthylisoquinoline alkaloid ancistrocladinium $A$, from Ancistrocladus cochinchinensis (Ancistrocladaceae) with improved antiprotozoal activities. Phytochemistry. 2011;72(1):89-93.

25. Oliveira GM, Masuda MO, Rocha NN, Schor N, Hooper CS, Araújo-Jorge TC, et al. Absence of Fas-L aggravates renal injury in acute Trypanosoma cruzi infection. Mem Inst Oswaldo Cruz. 2009;104(8):1063-71.

26. de Souza AP, Olivieri BP, de Castro SL, Araújo-Jorge TC. Enzymatic markers of heart lesion in mice infected with Trypanosoma cruzi and submitted to benznidazole chemotherapy. Parasitol Res. 2000;86(10):800-8.

27. Almeida IC, Gazzinelli RT. Proinflammatory activity of glycosylphosphatidylinositol anchors derived from Trypanosoma cruzi: structural and functional analyses. J Leukoc Biol. 2001;70(4):467-77.

28. dos Santos PV, Roffê E, Santiago HC, Torres RA, Marino AP, Paiva CN, et al. Prevalence of CD8(+)alpha beta T cells in Trypanosoma cruzi-elicited myocarditis is associated with acquisition of CD62L(Low)LFA-1(High)VLA-4(High) activation phenotype and expression of IFN-gammainducible adhesion and chemoattractant molecules. Microbes Infect. 2001;3(12):971-84.

29. de Oliveira GM, Yoshida N, Higa EM, Shenkman S, Alves M, Staquicini D, et al. Induction of proinflammatory cytokines and nitric oxide by Trypanosoma cruzi in renal cells. Parasitol Res. 2011;109(2):483-91.

30. Cardoso J, Soares MJ. In vitro effects of citral on trypanosoma cruzi metacyclogenesis. Mem Inst Oswaldo Cruz. 2010;105(8):1026-32.

Correspondencia: Juan Rojas Armas

Dirección: Av. Separadora Industrial 1018, Lima 3, Perú

Teléfono: (51) 997251044

Correo electrónico: jprojasarmas@yahoo.com 